\title{
Automatic Volumetric Segmentation of Encephalon by Combination of Axial, Coronal, and Sagittal Planes
}

\author{
Rodrigo Siega, Edson J. R. Justino, Jacques Facon, \\ Flavio Bortolozzi, Luiz R. Aguiar \\ Pontificia Universidade Catolica do Parana(PUCPR), \\ Curitiba, Parana, \\ Brazil \\ http://www .pucpr.br
}

\begin{abstract}
This paper describes a method of automatic volumetric segmentation of the human encephalon by Magnetic Resonance Imaging $(M R I)$ using three anatomical planes of visualization (axial, coronal and sagittal). For mapping the volumetric topography of the encephalon we developed a set of algorithms for managing the different planes. It is intended for the segmentation of magnetic resonance images with $T 1$ weighting, Inversion Recovery $(I R)$ and Gradient Echoes GRE (T1 IR $G R E)$. By combining filtering techniques and techniques of adaptive multiscale representation, directional transformation, and morphological filters, the method generates separated masks of the encephalon in the axial, coronal, and sagittal planes. Based on the masks of the three planes, reconstruction and rendering of the encephalon surface, which reveal the cortical mantle, are carried out. Tests performed using a database containing DICOM images of 30 volunteers show that the proposed method of automatic volumetric segmentation is promising for the study described in this paper.
\end{abstract}

Keywords: magnetic resonance imaging, brain, anatomical planes, $T 1$ IR GRE.

\section{Introduction}

The cerebral cortex is the outermost layer of the brain in vertebrates. It is replete with neurons and is the location where the most sophisticated and distinguished neuronal processing takes place (Figure 1) ${ }^{1}$.

The human cortex is 2 to $4 \mathrm{~mm}$ thick, with a planar area of approximately $0.22 \mathrm{~m}^{2}$. It plays the lead role in complex brain functions such as memory, attention, consciousness, language, pereption, and thought [12].

\footnotetext{
1 This image is a work of the National Institutes of Health, part of the United States Department of Health and Human Services. As a work of the U.S federal government, the image is in the public domain
} 
The cortical mantle is composed of hemispheres that have many convolutions. Each convolution is called a gyrus, and each depression between gyri is called a sulcus. Particularly, deep sulci may still be called fissures. This mechanism of using sulci and gyri is a way to increase the brain area without increasing its volume. Consequently, about two thirds of the surface is hidden.

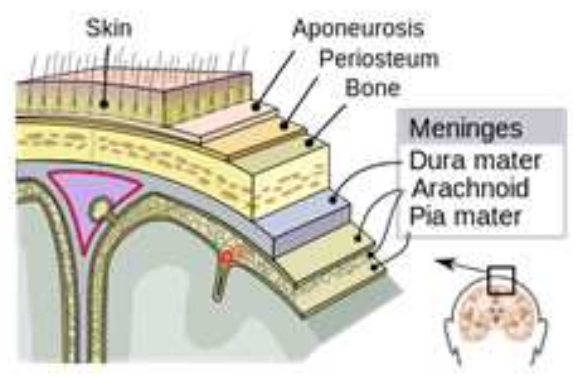

Fig. 1. Schematic diagram of different tissues involving the brain.

This study presents a method of automatic segmentation of the encephalon by $M R I$ with relaxation time ( $T 1)$, gradient echoes $(G R E)$ and inversion recovery (IR). The choice of this type of imaging was motivated by the lack of technical segmentation of the brain when using $M R I$ with inversion recovery (T1 IR $G R E$ ). Another innovative aspect of this work is the use of three visualization planes for $M R$ images (axial, coronal, and sagittal), providing a higher level of accuracy in the process in comparison with segmentation only by a plane. The proposed method is divided into four main stages.

First, pretreatment of the images in the three anatomical planes (axial, coronal, and sagittal) is carried out. After that, segmentation of the encephalon surface is carried out in three planes evenly and separately. Then, post-processing is carried out for, among other purposes, reconstructing the volumetric sagittal plane, based on the three anatomical planes. Finally, rendering of the surface of the encephalon, which reveals the cortical mantle, is carried out.

The rest of the work is organized as follows. First, the materials and methods used in this work are described in section 3. Section 4, describes the experiments and the discussion of the results.

\section{Background}

$M R I$ contrast is influenced by several characteristics of the tissues and other factors such as relaxation time $T 1, T 2$ and density of protons $P D$, as shown in Figure 2. The relaxation rates affect the signal-to-noise ratio $(S N R)$ of the image and are directly linked with the parameters of the signal, which are time of repetition $(T R)$ and echo time $(E T)$. 
These can be adjusted to highlight imaging contrast properties such as weighting [4]. Spin-echo $(S E)$ pulse sequences use radio frequency $(R F)$ excitation pulses of $90^{\circ}$ and $180^{\circ}$ to tilt the effective magnetization vector $(E M V)$ in transverse and inverted longitudinal planes, respectively.

The $G R E$-like pulse sequence uses a variable $R F$ pulse and tilts the $E M V$ to any angle, but not to $90^{\circ}$ or $180^{\circ}$. Exams conducted with $G R E$ pulse sequences are faster than those conducted with $S E$ pulse sequences. However, since there is no compensation for disturbances in field homogeneity, which are constant in $G R E$ sequences, they are more susceptible to the occurrence of unwanted artifacts [4]. Therefore, it is always important to use a filter to improve the signal-to-noise ratio.
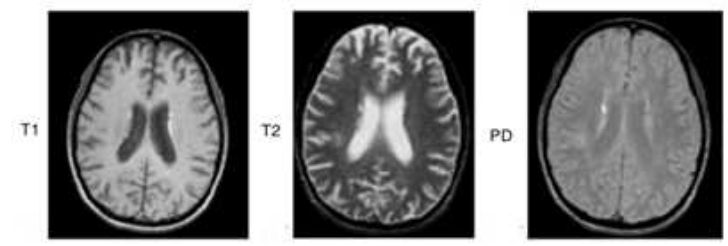

Fig. 2. Images in axial sections, SEpure,withweightingsinT $1(T E / T R=$ $14 / 500 \mathrm{~ms}), T 2(T E / T R=80 / 500 \mathrm{~ms})$, andPD $(T E / T R=30 / 500 \mathrm{~ms}) * \mathrm{~ms}$ (milliseconds).

Historically, $I R$ techniques were widely used in the early days of the $M R I$ (1980 1985). They produced excellent image contrast, especially for $T 1$-weighted images. However, they demanded too much time (usually 15 to $20 \mathrm{~min} /$ sequences). Subsequently, $S E$ techniques largely replaced $I R$ in most applications. Nonetheless, in the late 1990, a generation of fast $S E$ signals (FSE) allowed high quality $I R$-FSE sequences to be carried out over a more reasonable period ( 5 to $10 \mathrm{~min} /$ sequences). Today, $I R$ techniques are widely used in all branches of $M R I$, especially in neuroradiology and cardiac imaging [9].

$M R$ images of the brain using $T 1$ I $R$ GRE inversion recovery sequences with isotropic volumetric images have the advantage of highlighting the difference between gray and white tissue, and water and fat.

Such highlights may significantly improve the revealing of sulci and gyri, and are also useful for surgical procedures, and functional diagnostic studies $[2,9]$.

\section{Materials and Method}

The process of MRI segmentation of the encephalon, and consequently of the cortical mantle, poses numerous challenges. The presence of reduced thickness tissues, such as pia mater, turns the segmentation process into a delicate procedure, even for a specialist who intends to segment it using manual procedures. 


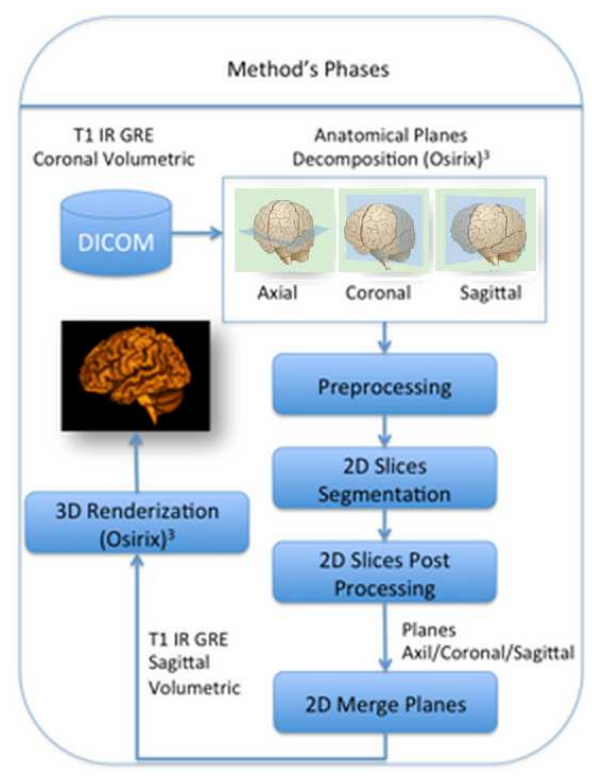

Fig. 3. Flow chart of proposed method.

The low resolution of the $M R I$ (between 128 and 512 pixels, typically) is among the factors that interfere with this process. This hinders the identification of more subtle tissues.

Specific segmentation procedures should be considered in these cases. Despite their complexity that characterized hybrid methods [6], these have the advantage of not taking only one approach, but associating the advantages of multiple techniques used at different stages of the process, to meet the processing needs of each tissue involved more effectively. Figure 3 shows the flow chart of the proposed method.

The diagram is divided into four main stages: preprocessing of images of the three anatomical planes (sagittal, axial, and coronal), segmentation of $2 D$ slices, post-treatment of segmented slices, and volumetric sagittal reconstruction. Two other steps are performed by the Osirix tool. The first one consists of a decomposition process of the slices in the three anatomical planes, and the second focuses on the rendering of the brain.

\subsection{MRI Database}

In this study, a database in the Digital Imaging and Communications in Medicine $(D I C O M)$ standard was employed for $M R I$. The $D I C O M$ were obtained in two volumetric sequences: $T 1 G R E$ and $T 1 I R$ GRE, with Magneton Symphony 1.5T Siemens equipment. The base is composed of 30 sets of DICOM files of adult volunteers, aged 25 years on average. Of these, 16 volunteers were women, and 14 volunteers were men. 
Automatic Volumetric Segmentation of Encephalon by Combination of Axial, Coronal, and ...

The collection of images was performed after approval by the Research Ethics Committee of $P U C P R^{2}$, protocol number 746,028. For this study, only $T 1 I R$ $G R E$ images were used.

\subsection{MRI Preprocessing}

During preprocessing, each image of the DICOM package (slices) is initially decomposed into the three anatomical visualization planes (axial, coronal, sagittal), according to Figure 3 .

Plane redundancy is used by the merge algorithm to generate the final volumetric plane. The generation of images in different planes was made using the Osirix tool ${ }^{3}$. At the end of the segmentation process, individual planes are grouped again to generate a new DICOM package with the volumetric plane. Any of the three planes can be used for reconstruction. In this study, the sagittal plane was chosen. Then, this plane may be rendered and displayed in a DICOM viewer, which for this study was Osirix.

The set of slices of each anatomical plane undergoes a sequence of filters with the aim to prepare it for the segmentation phase. Each step of the process is presented below.

Denoising. An important step in the preparation of $M R I$ is the attenuation of noise inherent in image acquisition. The $T 1 I R G R E$ weighting offers $T R=$ $4,000 \mathrm{~ms}$, which provides a high signal-to-noise $S N R$ ratio. Still, a procedure for noise reduction was adopted with the aim of minimizing the influence of the unwanted artifacts of subsequent steps. The most common acquisition method for obtaining $M R I$ is acquisition in the frequency domain [8]. The acquired signal $Y(\mu, v)$ may be decomposed into two components: the signal of interest $S(\mu, v)$ and noise $N(\mu, v)$, in horizontal $\mu$ and vertical $v$ frequencies, respectively:

$$
Y(\mu, v)=S(\mu, v)+N(\mu, v) .
$$

The noise $N(\mu, v)$ is a complex Gaussian white noise. This noise derives from the temperature of the tissue. Therefore, the structure of this noise depends on the signal $S(\mu, v)$. Thus, the noise in $M R I$ depends on the signal, whose properties are best described by a Rician distribution $[1,3]$.

In this study, the wavelets domain was chosen for the noise image filtering process $[5,11]$, since the processed discrete $2 D$ wavelets $(2 D-D W T)$ tend to concentrate the energy of the desired $S$ signal in a reduced set of coefficients. Therefore, the transformed wavelet of a noisy image consists of a small number of coefficients with high $S N R$, which is desirable. In addition, a high number of coefficients with low $S N R$ ratios are disposable. After excluding the coefficients with low $S N R s$, the image is reconstructed using the inverse wavelet transform.

\footnotetext{
${ }^{2}$ Pontifcia Universidade Catolica do Parana, Brazil.

${ }^{3}$ Osirix v.5.7.1, 32 bit / Free Version, Pixmeo Sarl.
} 
The challenge to be faced with regard to $M R I$ noise is to determine which factors have a high $S N R$ ratio.

For our purposes, the transform wavelet was used with a $H A A R$ base function with level -2 hard thresholding. The choice was based on the results already published in other works $[5,7,11]$.

Histogram Equalization. Histogram equalization was necessary in order to establish a better balance of gray levels, thus homogenizing the areas. This makes easier the removal of unwanted artifacts, which is typical of $G R E$ acquisition. Consider a set of $M R$ images $\phi_{i}$ in which i is a DICOM set, acquired in $T 1 I R$ $G R E$ in 256 gray levels.

The histogram in question highlights the region of interest (ROI), the brain, which is represented as a concentration of gray levels corresponding to approximately $25 \%$ of total 256 levels represented in the image. The histogram equalization in 25 levels enables the resizing of the level scale corresponding to the $R O I$. This procedure highlights the gray levels of the $R O I$ to the detriment of other regions.
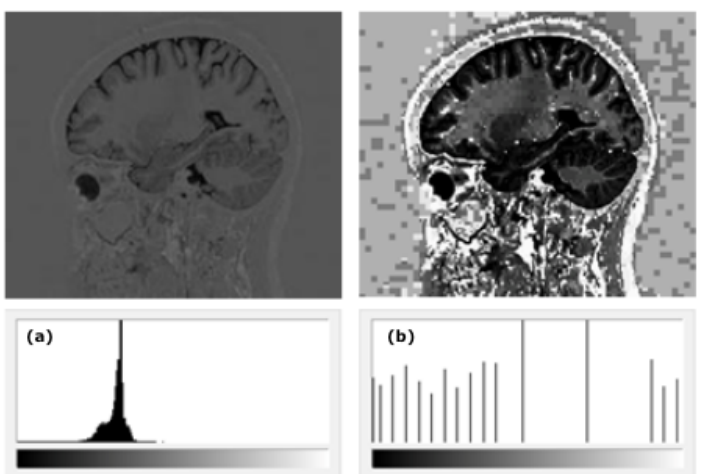

Fig. 4. (a) Image $T 1 I R G R E$ and its histogram, (b) image equalized and its histogram.

Grayscale Morphological Reconstruction. Morphological reconstruction in gray levels aims to reestablish the connectivity between adjacent pixels and to attenuate the influence of artifacts typical of GRE acquisition. The process occurs in a controlled manner through successive expansions [7]. The control of geodesic dilatation is accomplished through an image marker $\phi_{i}$, that is, the desired image. This is carried out under a base image $g$, which is an image with a black background and edges from the image $\phi_{i}$ in this case 2 :

$$
\phi_{i}^{\prime} \leftarrow g^{T^{i}} D^{\phi_{i}} \leftarrow\left(\phi_{i} \oplus_{g} D\right)^{\infty} .
$$


Then, a nonlinear median filter was applied with the aim of attenuating the noise resulting from the reconstruction step.

It is very effective in eliminating image impulse noises (Poisson noise) and preserving edges without introducing new values of gray levels in the image [13]. The nonlinear median filter chooses the element in a median position on the ordered list of values of gray levels in a window (usually $3 \times 3$ ) around the center pixel.

Outline Smoothing. Candes and Donoho [5] developed a multiscale transform called a curvelet. This transform was designed to represent edges and other singularities along curves, and is much more efficient than traditional transforms. The curvelet transform, as well as the wavelet transform, has indexed elements of structure by parameters of scale and location. Unlike the wavelet transform, the curvelet has directional parameters, and the curvelet pyramid contains elements with a high degree of directional specificity. In addition, it is anisotropic in scale, unlike the wavelet. In this study, the Fast Discrete Curvelet Transform (FDCT) and the inverse (IFDCT) [13] were used to remove possible discontinuities and unwanted noise in the contour region of the $R O I$.

In this study, $2 D F D C T$ by wrapping was used, since it is the simplest transform. It has low redundancy, and it is quick. The most significant $10 \%$ coefficients were used, thus eliminating small edge distortions, and for smoothing out the variability of the bottom of the image [13].

\section{3 $2 D$ Slice Segmentation}

In order to separate the $R O I$ from the rest of the slice image, a mask was created by the binarization of images $\delta_{i}$ of each slice $i$. The binarization process for each slice took into consideration the different tissues constituting the brain, and the position of the slice of the DICOM set. The most central region of the brain is composed of white tissue (predominantly gray levels closer to white) and a peripheral region composed of gray tissue (predominantly shades of gray closer to black). Each slice was treated according to mean indexes $\beta$ observed in a range consisting of a set of slices, as shown in Figure 5 . The values were obtained by analyzing samples from six different volunteers. The binarization algorithm is based on the average values in the three planes, considering similarities in the variability of thresholds. The binarization process by a fixed threshold in $\beta$ of the image $\delta_{i}$ occurs in each slice in the three different planes. The $\beta$ value represents the percentage of normalized gray levels between 0 (white) and 1 (black). Finally, a process of reversing the image takes place to keep black the background 3 :

$$
\delta_{i}^{\prime} \leftarrow \text { inverse }\left\{\text { treshold }\left(\left(\beta, \delta_{i}\right) \mid 0 \leq \beta \leq 1\right\} .\right.
$$

ROI Mask Selection. After the binarization process, an algorithm for filling the area with a closed contour was applied. Therefore, many $\lambda_{\Theta}$ candidate regions 


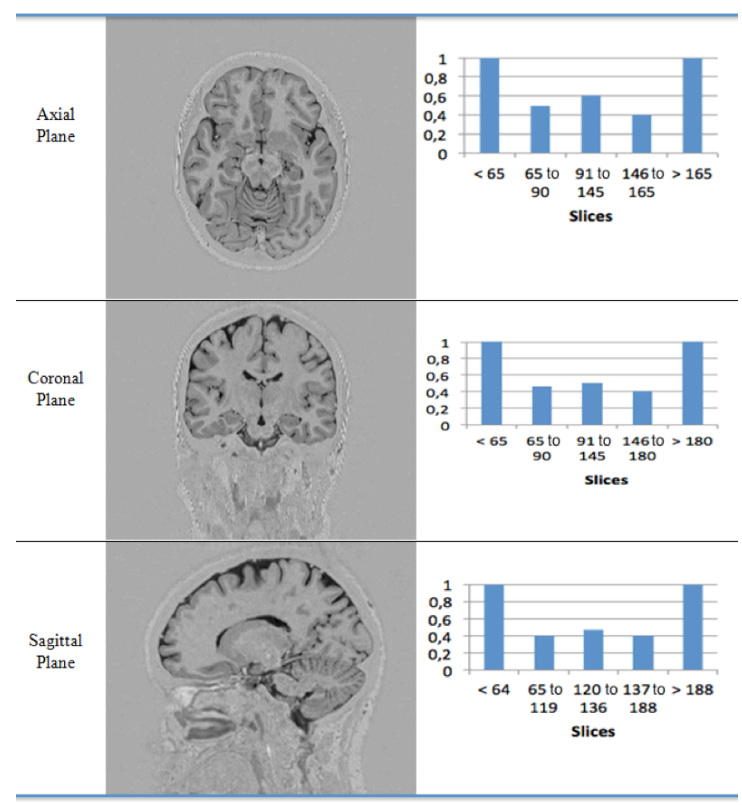

Fig. 5. Gray-level variations in $T 1 I R G R E$ images from three planes (image brightness is $+50 \%$ for better viewing).

to mask the $R O I$ of $i$ were formed. The selection of a candidate was made using the selection of the greater area of set:

$$
R O I_{i} \leftarrow \max \left\{\lambda_{\Theta} \mid \Theta \in \mathbb{N}\right\} .
$$

\section{4 $2 D$ Slice Post-Processing}

From the mask $R O I_{i}$, a combination of the original image $\phi_{i}$ may be performed. Consequently, ROI may be delimited. However, in this first stage of segmentation, it was not possible to highlight regions of sulci and gyri in the cortical mantle adequately. The region in question is known as subarachnoid space and involves the entire surface of the brain, Figure 1.

To remove the subarachnoid space, repetition of three previously mentioned steps in the process is necessary. The steps are as follows: thresholding with cutoff obtained by the algorithm of [10] with the aim of removing the subarachnoid space, application of a $3 \times 3$ median filter for smoothing the contour, and the selection of the greater area for the removal of unwanted artifacts that persist, as shown in Figure 6 . The new mask $R O I_{i}^{\prime}$ is ready to be merged between anatomical planes $\phi_{i}$. 


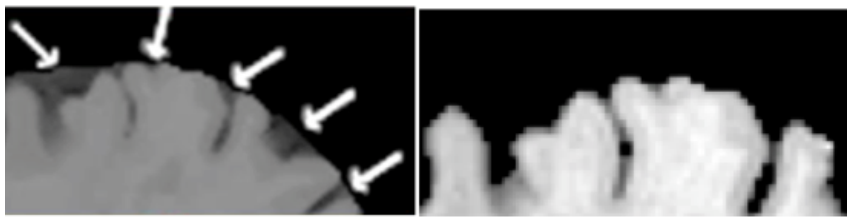

Fig. 6. Post-processing result.

\subsection{D Merge Planes}

The process of pairing is a pixel to pixel combination between images of the axial, coronal, and sagittal planes after slice-by-slice segmentation, Figure 7. This is carried out to generate an image whose area is more likely to belong to the encepholon region. Reconstruction of the volumetric sagittal plane is then achieved by the combination of the three anatomical segmented planes. Reconstruction is based on the principle of decision by majority vote. That is, each pixel viewed in the sagittal plane image is identified in the three anatomical planes. If the pixel is identified as belonging to the $R O I$ in at least two of them, it is maintained in the volumetric plane. Otherwise, it is eliminated. Given that the central slices of each anatomical plane are less susceptible to undesirable content in comparison with the edges, a weight is given to each slice, decreasing from the central position to the periphery. If there is only one anatomical plane, this indicates that the pixel belongs to the $R O I$, and an assessment of the weight of this slice is carried out before disposing it. If the weight of this slice, receiving a positive result, is greater than the other two, the pixel is maintained.

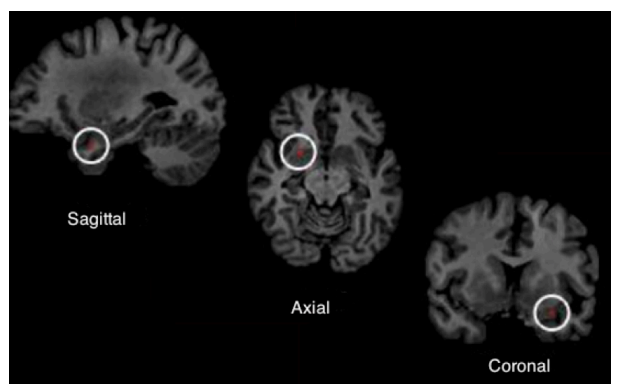

Fig. 7. The vision of the same pixel in the three planes of projection.

\section{6 $3 D$ Renderization}

A rendering of the volumetric sagittal plane, resulting from the merge algorithm between the three anatomical planes, can be read by a DICOM standard visualization tool. In this study, the Osirix tool was used. Figure 8 presents 
the results for a set of volunteers from the database. The volumetric images, seen in the three anatomical planes, emphasizing the presence of the sulcus and gyrus of the cortical mantle.

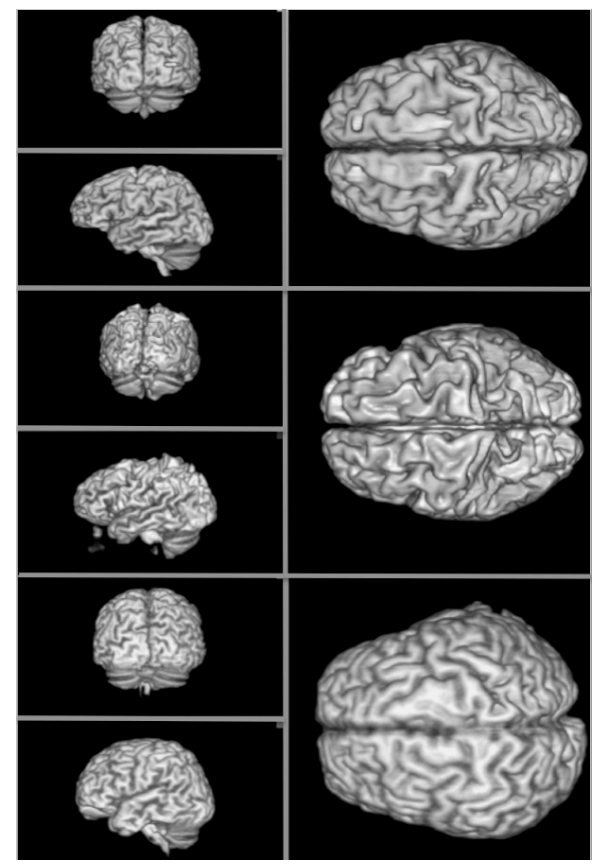

Fig. 8. The view of the cortical mantle on the three viewing planes.

\section{Experiments and Analysis}

Evaluating the results of the segmentation proved to be a laborious task. The three anatomical planes were processed. Considering that each plan has 250 images (slices) on average, generating a perfect image (ground truth) of each segmented slice proved to be impractical. Another possible solution was to seek an available tool to perform this type of comparison. However, owing to the use of T1-type images with recovery of $T 1 I R G R E$ gradient-echo inversion, which is unprecedented, no tool was found. This also represents one of the contributions of this study. Thus, the assessment was visual and was performed as follows. Each image segmented by the proposed method was examined visually, by a field specialist. If the image displayed artifacts resulting from the method, segmentation of the image would be deemed erroneous.

The visual assessment suggested that the method was promising. It had an exact segmentation rate in $80 \%$ of the slices. As mentioned in the introduction 
of this paper, the processes of segmentation are strongly linked to weights used in the acquisition of the slices, which does not guarantee flexibility in this regard. The similarity between texture tissue and the degree of gray levels is another factor that affects the efficiency of the method. We observed that the proposed method did not always succeed in segmentation. It displayed artifacts originating from inadequate segmentation. Furthermore, in other cases, it had failures resulting from the improper disposal of certain tissues.

Figure 9 depits some examples. The majority of problems occurred in a slice or in a reduced group of slices. Nevertheless, this affects not only the quality of slice segmentation but also the rendering process, incorporating nonexistent artifacts into the cortical mantle. As previously mentioned, the method was promising, even though it requires in depth studies to improve not only the steps of segmentation but also the plane merge algorithm.

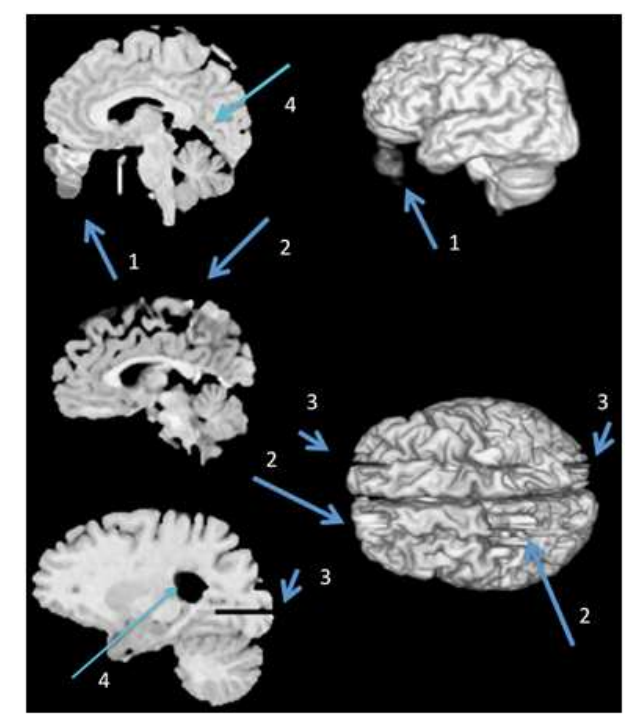

Fig. 9. Examples: (1) slice with artifacts resulting from inadequate segmentation, (2, 3 , and 4) slices with inadequate removal of tissue

\section{Conclusion and Future Works}

This paper presented an automatic volumetric segmentation algorithm for the encephalon. It used $T 1$ type magnetic resonance images with recovery of inverted $I R$, gradient echoes $G R E$ and involving the three anatomical planes of visualization (axial, coronal, and sagittal). The use of a complex but reproducible sequence of processes (using techniques such as denoising, contrast enhancement, 
morphological reconstruction, outline smoothing, and slice binarization) to generate the encephalon mask in three axial, coronal, and sagittal planes separately proved to be efficient. This enabled the reconstruction and rendering of the encephalon surface with high precision. Experiments performed in a database with 30 volunteers showed an average rate of $80 \%$ in slices segmentation of each $D I C O M$ set belonging to the database.

The first contribution of this work is the use $M R I$ in $T 1 I R G R E$ acquisition, which was of paramount importance for obtaining such results.

Another contribution is the use of three visualization plans of anatomic projection. Using the three plans, there is the possibility of an additional plan complementing the results of the others when the region of interest does not have appropriate segmentation. Consequently, this provides a greater level of accuracy for the process in comparison with segmentation by a single plan.

A third contribution from a computational point of view is the combination of several techniques that provided an innovative method. This makes it possible for studies on anatomical patterns of the encephalon to be conducted with greater confidence and agility. These techniques display more detailed images than the slices analyzed in a single plane, and they facilitate obtaining images of patients by automated means of segmentation.

However, despite the benefits of using this type of image, the algorithm was not able to segment all slices properly, maintaining artifacts in some slices and removing part of the tissue in others. This is an indication of the size of the challenge to be faced and overcome.

In our future work, we intend to perfect both the segmentation and the merge algorithms, with the aim of correcting the mistakes found.

\section{References}

1. A., A.: Wavelet methods in statistics: Some recent developments and their applications. Statistics Surveys 1, 16-55 (2007)

2. A., M.: Noise in mri. Magn Reson Med 36(3), 494-497 (1996)

3. Abramovich F., Bailey T. C., S.T.: Wavelet analysis and its statistical applications. Journal of the Royal Statistical Society Series 49(6810), 1-29 (2000)

4. B., G.C.: Physical Principles of Computed Tomography and Magnetic Resonance Imaging, vol. 1. Williams and Wilkins, 2 edn. (2000)

5. Candes E. J., D.D.I.: Curvelets: A surprisingly effective non adaptive representation for objects with edge. Vanderbilt Univ. Press 1, 105-120 (2000)

6. Despotovic I., Goossens B., P.W.: Mri segmentation of the human brain: Challengers, methods, and applications. computational and mathematical methods in medicine. Computational and Mathematical Methods in Medicine 49(45341), 23 (2015)

7. Dougherty E. R., L.R.A.: Hands-On Morphological Image Processing, Tutorial Texts in Optical Engineering, vol. TT59. SPIE Press, 1 edn. (2003)

8. Kim S., Richter W., U.K.: Limitations of temporal resolution in functional mri. Magn Reson Med 37(1), 631-636 (1997)

9. Mancall E., B.D.: Gray's Clinical Neuroanatomy: The Anatomic Basis for Clinical Neuroscience, vol. 1. Elsevier Saunders, 1 edn. (2010) 
Automatic Volumetric Segmentation of Encephalon by Combination of Axial, Coronal, and ...

10. N., O.: A threshold selection method from gray-level histograms. IEEE Transactions on Systems, Man and Cybernetics 1(SMC-9), 62-66 (1979)

11. R., N.: Wavelet-based rician noise removal for magnetic resonance imaging. IEEE Transactions on Image Processing 8(10), 1408-1419 (1999)

12. Tamraz J.C., C.Y.: Atlas of Regional Anatomy of the Brain Using MRI - With Functional Correlation, vol. 1. Springer-Verilag, 1 edn. (2006)

13. Xiaojun W., L.W.: Ct/mri medical image based on sub-band coefficients selection through curvelet transform method. Journal of Chemical and Pharmaceutical Research 6(3), 1083-1088 (2014) 